\title{
POLÍMEROS NA OBTENÇÃO DE SISTEMAS DE LIBERAÇÃO DE FÁRMACOS
}

\author{
POLYMERS IN DRUG DELIVERY SYSTEMS \\ Oliveira R. B. ${ }^{1,2}$; Lima E. M. ${ }^{1, *}$ \\ ${ }^{1}$ Laboratório de Tecnologia Farmacêutica - Faculdade de Farmácia - Universidade Federal de Goiás - Brasil \\ ${ }^{2}$ Mestrando em Química, Instituto de Química-Universidade Federal de Goiás - Brasil
}

Recebido em 16/05/2006 - Aceito em 04/07/2006

* Autor para correspondência: emlima@farmacia.ufg.br

RESUMO: Devido as suas diversas aplicações e funcionalidades, especialmente em terapias de liberação controlada de fármacos, os polímeros estão dentre os excipientes mais utilizados em tecnologia farmacêutica. A escolha de um polímero para um sistema de liberação de fármacos depende de vários fatores que correlacionam as propriedades do princípio ativo, dos polímeros e demais excipientes que irão fazer parte da formulação. Além disso, o mecanismo pretendido de liberação do fármaco também interfere nessa escolha. Para se chegar ao mecanismo desejado de liberação do fármaco, os polímeros (sintéticos) podem ter suas propriedades moldadas ou pode-se trabalhar com uma mistura de polímeros que apresentam propriedades diversas e, com a modificação da proporção destes na formulação, pode-se chegar ao sistema de liberação que satisfaça às necessidades do formulador. Avanços futuros na ciência dos polímeros deverão ser baseados em modificações de suas propriedades químicas e físicas, além de novas combinações de copolímeros com objetivos e componentes capazes de liberar uma ampla variedade de agentes bioativos de forma programada e controlada.

PALAVRAS-CHAVE: Polímeros, sistemas de liberação de fármacos.

ABSTRACT: Due to their broad range of applications, particularly for the controlled release of drugs, polymers are among the most widely used excipients in pharmaceutical technology. Selecting a polymer for a drug delivery system depends on its characteristics, as well as the properties of the drug substance and of the remaining ingredients of the formulation. Selecting a polymer is also influenced by the desired mechanism for drug release. To achieve specific drug release profiles, synthetic polymers may be chemically modified. Also, blending different polymers at different ratios may lead to specific drug release systems. Future developments in polymer sciences will be based on modifications of the chemical and physical properties of the polymers and on new combinations of copolymers able to provide a programmed and controlled release of a wide variety of drugs.

KEYWORDS: Polymers, drug delivery systems.

\section{INTRODUÇÃO}

A vida é polimérica na sua essência: os mais importantes componentes de uma célula viva (proteínas, carboidratos e ácidos nucléicos) são todos polímeros. A natureza usa os polímeros para construção e como parte do complicado mecanismo celular (GALAEV; MATTIASSON, 1999). Os polímeros são uma das classes de materiais mais versáteis e têm mudado nosso cotidiano por várias décadas (PILLAI; PANCHAGNULA, 2001) com importantes aplicações na área médica, agricultura (CHANDRA; RUSTGI, 1998) e engenharia (LANGER; PEPPAS, 2003). A fusão da ciência de polímeros com as ciências farmacêuticas conduziu para um avanço espetacular em termos de "inovação" (flexibilidade no estado físico, forma, tamanho e superfície) no design e desenvolvimento de novos sistemas de liberação de fármacos (SLFs) (PILLAI; PANCHAGNULA, 2001).

Um sistema polimérico de liberação de fármaco por via oral pode ser planejado e desenvolvido por vários métodos de processamento. Modificando as propriedades do polímero, um sistema de matriz pode ser elaborado para uma liberação sustentada ou controlada do fármaco. Materiais poliméricos farmacêuticos com pequena ou nenhuma toxicidade podem ser utilizados como membranas ou matrizes nas quais o ingrediente ativo é disperso 
Oliveira, R.B./Revista Eletrônica de Farmácia Vol 3 (2), 29-35, 2006.

ou dissolvido. Polímeros também funcionam como veículos e podem ser adicionados aos ingredientes ativos. Esses carreadores podem ser usados para liberar uma grande variedade de fármacos em uma taxa controlada no trato gastrintestinal (ZHU, 2002).

Devido as suas diversas aplicações e funcionalidades, especialmente em terapias de liberação controlada do fármaco, os polímeros estão dentre os excipientes mais utilizados para a obtenção de formas farmacêuticas (RIOS, 2005).

\section{CRITÉRIOS PARA A SELEÇÃO DE POLÍMEROS}

Os polímeros, e também todos os outros excipientes, são selecionados de acordo com a formulação e o mecanismo de liberação pretendido, como por exemplo forma de dosagem parenteral ou entérica. Porque suas atividades dependem bastante das demandas e tendências dentro das indústrias farmacêuticas, os fabricantes de polímeros estão muito atentos aos fatores que direcionam as decisões de seus clientes (RIOS, 2005), ou seja, procuram proporcionar ao polímero propriedades que permitam a sua aplicabilidade em determinadas formulações. Um exemplo atual é o maior interesse das indústrias farmacêuticas em busca de polímeros de base aquosa em substituição aos que necessitam de solventes orgânicos para sua produção ou aplicação. Problemas como falta de biocompatibilidade, solventes residuais e efeitos danosos em fármacos incorporados durante a fabricação da formulação ou durante a erosão do polímero depois da aplicação são descritos (SCHWENDEMAN et al., 1996) e rejeitados pelas indústrias farmacêuticas. Portanto, a função, segurança da formulação e também a melhor posologia para o paciente são fatores que devem ser levados em consideração na seleção do polímero (RIOS, 2005).

Alguns fármacos que apresentam baixa biodisponibilidade, ou quando a substância ativa tem um efeito colateral de irritação local podem ter esses problemas solucionados quando preparados em formulações multiparticuladas com matriz polimérica que proporciona uma liberação controlada (PALMIERI et al., 2002; SJOBLOM, 2004). Quando uma mistura consistindo de um fármaco pouco solúvel em água e um veículo inerte, altamente solúvel, é dissolvida em um meio aquoso, o veículo se dissolve rapidamente liberando cristais muito finos do fármaco. A extensa área de superfície resulta em um aumento da taxa de dissolução e portanto melhora a biodisponibilidade (ZHU, 2002). Logo, as propriedades físico-químicas do princípio ativo, principalmente solubilidade e permeabilidade (ZHU, 2002), e como ele pode ser melhor absorvido pelo organismo também tem bastante importância para a escolha do polímero da formulação (RIOS, 2005). Os sistemas de liberação poliméricos são principalmente aplicados para se conseguir um controle temporal ou espacial da liberação do fármaco (LI, 1999) e são ferramentas de grande importância para tecnologia farmacêutica.

Além da função, ou objetivos da formulação e das características do princípio ativo, as propriedades físicoquímicas do polímero são fatores de suma importância que determinam sua utilização (PILLAl; PANCHAGNULA, 2001; RIOS, 2005). Essas propriedades são dependentes da natureza química dos monômeros, do processo e da técnica de preparação do polímero, do peso molecular, que depende das condições de polimerização para cada monômero, e da estrutura macromolecular (MANO; MENDES, 1999). As propriedades físicas dos polímeros estão relacionadas à resistência das ligações covalentes, à rigidez dos segmentos na cadeia polimérica e à resistência das forças intermoleculares (MANO; MENDES, 1999). Devido à diversidade inerente das estruturas e exigência do completo entendimento da superfície e propriedades do polímero que podem fornecer as funções químicas, interfacial, mecânica e biológica desejada, a seleção e design de um polímero se tornam uma tarefa bastante desafiadora (PILLAI; PANCHAGNULA, 2001).

Em relação às propriedades dos polímeros, dois critérios devem ser seguidos na elaboração de uma formulação. Em primeiro lugar, as características químicas do polímero não devem comprometer a ação dos ingredientes ativos; em segundo, as propriedades físicas do polímero devem ser consistentes e reprodutíveis de lote a lote. Depois desses critérios terem sido encontrados, os formuladores devem concentrar-se nas características específicas dos polímeros (RIOS, 2005). Dentre as várias propriedades dos polímeros, algumas se demonstram mais importantes quando na elaboração de um sistema de liberação de fármacos, como a permeabilidade (JACOBS; MASON, 1993), propriedades de superfície como hidrofilicidade, lubrificação, lisura, energia de superfície (ANGELOVA; HUNKELER, 1999), adesão, solubilidade (PILLAl; PANCHAGNULA, 2001) e temperatura de transição vítrea (ZHU, 2002).

Muitas classes de polímeros farmacêuticos têm sido usadas em sistemas de liberação controlada de fármacos devido as suas diferentes permeabilidades (JACOBS; MASON, 1993). Os Materiais mais comuns incluem hidroxipropil metilcelulose (HPMC), etilcelulose (EC), polivinilpirrolidona (PVP), e ésteres poliacrílicos. Estes materiais podem ser usados tanto para matriz quanto para revestimento na liberação controlada do fármaco (ZHU, 2002). A permeabilidade de um fármaco através de um material polimérico é um processo de três partes envolvendo dissolução, migração e difusão das moléculas do fármaco em função da solubilidade e coeficiente de difusão (ZHU, 2002). A estrutura cristalina de um polímero tem maior grau de empacotamento molecular e, portanto, tende a resistir mais à difusão de um fármaco em relação a polímeros amorfos com a mesma estrutura química (ZHU, 2002).

As propriedades de superfície como hidrofilicidade, lubricidade, uniformidade e energia de superfície determinam a biocompatibilidade com tecidos e sangue, além das propriedades físicas influentes como 
durabilidade, permeabilidade e degradabilidade (ANGELOVA; HUNKELER, 1999). As propriedades de superfície também determinam a capacidade de sorção de água de polímeros, que sofrem degradação hidrolítica e intumescimento (hidrogéis). Por outro lado, materiais usados por longo tempo (implantes ortopédicos e dentários) devem ser repelentes à água para evitar degradação ou processo de erosão que conduz para modificações na dureza e menor resistência mecânica. Propriedades de superfície podem ser melhoradas por meios químicos, físicos e biológicos para aumentar sua biocompatibilidade. O acoplamento de enzimas, fármacos, proteínas e anticorpos na superfície de polímeros tem originado "polímeros terapêuticos" para atingir órgãos e células (BROCCHINI; DUNCAN, 1999).

As propriedades de dissolução do polímero afetam bastante a liberação de princípios ativos (ZHU, 2002). Como já foi dito anteriormente, a dissolução do fármaco pode ser acelerada ou retardada dependendo da solubilidade ou permeabilidade do veículo (ZHU, 2002).

Para alguns processos de produção como a granulação via extrusão por fusão a quente (onde o polímero é fundido e funciona como um ligante e/ou agente retardador da liberação do fármaco), os polímeros que serão selecionados devem possibilitar o processo em temperaturas relativamente baixas em virtude da sensibilidade térmica de muitos fármacos. Isso significa que o polímero utilizado deve apresentar uma baixa temperatura de transição vítrea. Polímeros como HPMC, EC e PVP possuem altas temperaturas de transição vítrea limitando sua utilização. Já os polímeros acrílicos são amorfos e apresentam baixa temperatura de transição vítrea. A escolha do polímero também depende de sua processabilidade e estabilidade térmica (ZHU, 2002).

Apesar da escolha ser determinada, principalmente, de acordo com o princípio ativo, muito raramente se encontra um fármaco que não é compatível com as classes gerais de polímeros (RIOS, 2005). Porém, casos de incompatibilidade podem ocorrer. A interação de excipientes e fármacos influencia no mecanismo de desintegração, liberação, absorção e biodisponibilidade do fármaco (JACKSON et al., 2000). O fenômeno de adsorção é um exemplo da interação fármaco e excipiente. A adsorção física é devido a interações eletrostáticas, ligações hidrogênio ou forças de van der Waals e usualmente é reversível; enquanto na adsorção química, o adsorvido é ligado ao adsorvente por ligações químicas primárias, incluindo troca iônica, protonação e complexação, e é irreversível (JENQUIN; MCGINITY, 1994). A extensão da adsorção depende das propriedades físico-químicas do fármaco e do excipiente (ZHU, 2002). Eudragit ${ }^{\circledR}$ RS (ácido acrilico e metacrilico, baixa permeabilidade à água) e Eudragit ${ }^{\circledR} \mathrm{RL}$ (ácido acrilico e metacrilico, alta permeabilidade à água) são copolímeros que apresentam poucos grupos amônio quaternário. A presença de ambas as ligações reversível e irreversível entre ácido salićlico e Eudragit ${ }^{\circledR} \mathrm{RL}$ foi descrita, sugerindo mais do que um tipo de ligações de interação (JENQUIN; MCGINITY, 1994). O perfil de liberação do fármaco foi diretamente correlacionado com a interação fármaco-polímero (ZHU, 2002).

Dependendo do mecanismo de liberação, $\mathrm{o} \mathrm{pH}$ do polímero também pode ser uma importante propriedade. Quando o fármaco deve ser liberado em um $\mathrm{pH}$ específico (ou seja, no trato gastrintestinal ou no colon), polímeros não iônicos não podem ser usados porque eles são pH-independentes. Para alguns comprimidos revestidos o pH é neutro para evitar a interação entre o polímero e o fármaco. Em outras aplicações, a liberação mais uniforme do fármaco por todo trato gastrintestinal, que apresenta valores de $\mathrm{pH}$ diferentes dependendo da localização, é favorecida (RIOS, 2005).

\section{POLÍMEROS BIODEGRADÁVEIS E NÃO DEGRADÁVEIS}

Polímeros biodegradáveis vêm ganhando destaque ultimamente. Um vasto número de polímeros biodegradáveis vem sendo sintetizado e os microrganismos e enzimas capazes de degradá-los também estão sendo identificados. Nos países em desenvolvimento, a poluição ambiental por polímeros sintéticos tem provocado uma série de danos. Como resultado, esforços têm sido feitos para resolver estes problemas acrescentando aos polímeros a biodegradabilidade através de pequenas modificações nas suas estruturas (CHANDRA; RUSTGI, 1998).

A Tabela 1 traz uma lista representativa de polímeros que têm sido investigados para aplicações em liberação de fármacos e podem ser amplamente classificados em biodegradáveis e não-biodegradáveis. 
Tabela 1. Lista representativa dos polímeros usados em sistemas de liberação de fármacos.

\begin{tabular}{|c|c|}
\hline Classificação & Polímero \\
\hline \multicolumn{2}{|l|}{ Polímeros naturais } \\
\hline Polímeros a base de proteínas & Colágeno, albumina, gelatina \\
\hline Polissacarídeos & $\begin{array}{l}\text { Agarose, alginato, carragenina, ácido } \\
\text { hialurônico, dextran, quitosana, } \\
\text { ciclodextrinas }\end{array}$ \\
\hline \multicolumn{2}{|l|}{$\begin{array}{l}\text { Polímeros sintéticos } \\
\text { Biodegradáveis }\end{array}$} \\
\hline Poliéster & $\begin{array}{l}\text { Poli(ácido láctico), poli(ácido glicólico), } \\
\text { poli(hidroxibutirato), poli(e-caprolactona), } \\
\text { poli(ácido } \beta \text {-málico), poli(dioxanonas) }\end{array}$ \\
\hline Polianidrido & $\begin{array}{l}\text { Poli(ácido sebácico), poli(ácido adípico), } \\
\text { poli(ácido terftálico) e vários copolímeros }\end{array}$ \\
\hline Poliamidas & Poli(imino carbonatos), poliaminoácidos \\
\hline Polímeros fosforosos & Polifosfatos, polifosfonatos, polifosfazenos \\
\hline Outros & $\begin{array}{l}\text { Poli(ciano acrilatos), poliuretanos, éster } \\
\text { poliorto, Polidihidropirans, poliacetais }\end{array}$ \\
\hline \multicolumn{2}{|l|}{ Não biodegradáveis } \\
\hline Derivados de celulose & $\begin{array}{l}\text { Carboximetil celulose, etilcelulose, } \\
\text { celulose acetato, celulose acetato } \\
\text { propionato, hidroxipropil metilcelulose }\end{array}$ \\
\hline Silicones & Polidimetilsiloxano, sílica coloidal \\
\hline Polímeros Acrílicos & $\begin{array}{l}\text { Polimetracrilatos, poli(metilmetacrilato), } \\
\text { poli hidro(etilmetacrilato) }\end{array}$ \\
\hline Outros & $\begin{array}{l}\text { Polivinilpirrolidona, etilvinilacetato, } \\
\text { poloxameros, poloxaminas }\end{array}$ \\
\hline
\end{tabular}

A biodegradação é um processo natural pelo qual produtos químicos orgânicos no ambiente são convertidos a componentes simples, mineralizado e redistribuído por meio dos ciclos elementares como o do carbono, nitrogênio e enxofre (CHANDRA; RUSTGI, 1998).

Com respeito aos polímeros biodegradáveis, é essencial reconhecer que a degradação é um processo químico e a erosão é um fenômeno físico dependente dos processos de difusão e dissolução (PILLAl; PANCHAGNULA, 2001). A biodegradabilidade pode ser manipulada pela incorporação de uma variedade de grupos instáveis como éster, ortoester, anidrido, carbonato, amida, uréia e uretano na cadeia principal (MAO et al., 1999), e pode ocorrer por meio enzimático, químico ou microbiano. Essas vias de degradação podem ocorrer separadamente ou de forma simultânea e são muitas vezes influenciadas pelos seguintes fatores: estrutura química e composição do polímero, fatores físico-químicos (carga iônica, força iônica e pH), fatores físicos (forma, tamanho, defeitos na cadeia), morfologia (amorfos, semi-cristalino, cristalino, microestrutura, stress residual), mecanismo de degradação (enzimático, hidrólise, microbiano), distribuição de peso molecular, rota de administração e sítio de ação (PILLAI; PANCHAGNULA, 2001).

Polímeros naturais (ou biopolímeros) são usualmente biodegradáveis e oferecem excelente biocompatibilidade, mas sofrem variação de lote a lote devido às dificuldades na purificação. Por outro lado, polímeros sintéticos estão disponíveis em uma extensa variedade de composições com fácil ajuste das propriedades (ANGELOVA; HUNKELER, 1999). A Figura 1 mostra a estrutura química dos principais biopolímeros utilizados em SLF. 


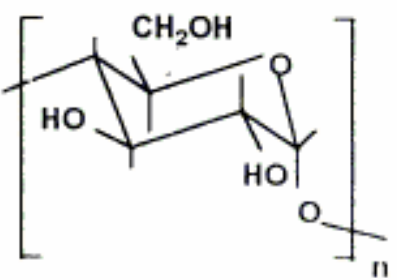

Amido

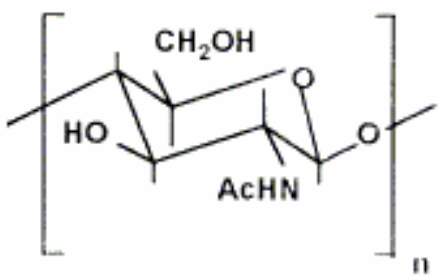

Quitina

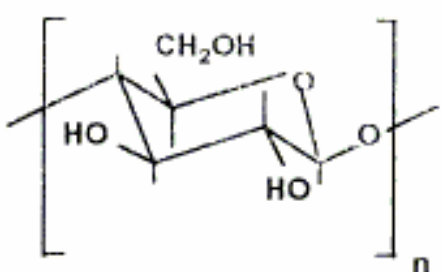

Celulose

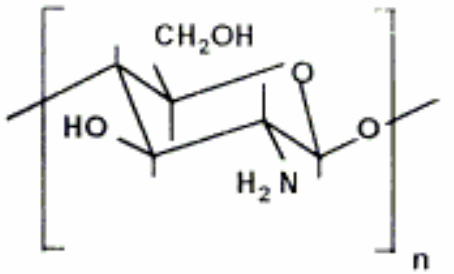

Quitosana

Figura 1. Estruturas de polissacarídeos (CHANDRA; RUSTGI, 1998).

Como exemplo de polímeros sintéticos biodegradáveis estão os poliésteres poli(ácido láctico) e poli(ácido glicólico). São polímeros de grande utilização na área farmacêutica. Fazem parte do grupo de polímeros que possuem grupos hidrolisáveis em suas cadeias (CHANDRA; RUSTGI, 1998), os quais são susceptíveis a biodegradação (ANGELOVA; HUNKELER, 1999). A Figura 2 mostra a estrutura do poli(ácido glicólico) (PGA) e do copolímero poli(ácido lático-co-glicólico) (PGA/LA).

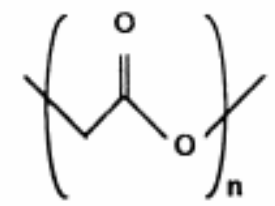

GA

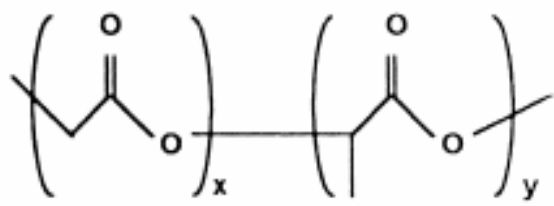

GA
LA

\section{poli(ácido glicólico) - PGA poli(ácido lático-co-glicólico) - PGALA}

Figura 2. Polímeros com cadeia hidrolisável, poli(ácido glicólico) e o copolímero poli(ácido lático-co-glicólico) (CHANDRA; RUSTGI, 1998).

É possível modificar as propriedades mecânicas, térmicas e biológicas do poli(ácido lático) (PLA) pela alteração de sua estereoquímica. Além disso, a biodegradabilidade pode ser ajustada modificando-se a proporção de PLA e PGA no copolímero. Apesar do PGA/LA representar o "modelo de ouro" dos polímeros biodegradáveis, a acidez local aumentada em razão da degradação pode provocar irritação no local de aplicação do polímero (UHRICH et al., 1999). Além disso, a acidez local aumentada pode também ser prejudicial à estabilidade de fármacos protéicos (FU et al., 2000).

Uma variedade de polímeros não biodegradáveis é usada na liberação de fármacos, dos quais os polímeros derivados de celulose e acrílicos encontram vasta aplicação na fabricação de formas de dosagem peroral, filmes transdérmicos e outros dispositivos (KUMAR; KUMAR, 2001). A misturas de polímeros com propriedades diferentes permitem um ajuste das formulações para o maior controle na liberação do fármaco. Lecomte et al. (2003) utilizou etilcelulose (EC) (polímero insolúvel) misturado ao copolímero ácido metacrilato- etil acrilato (Eudragit ${ }^{\circledR}$ L) (polímero entérico) para revestimento de formas farmacêuticas multiparticuladas com o propósito de moldar a liberação do fármaco em valores de $\mathrm{pH}$ altos e baixos variando-se na mistura a proporção dos polímeros. Um clássico exemplo da combinação de dois diferentes polímeros para o revestimento com filme é o uso da mistura a base de etilcelulose e HPMC. Em contraste ao EC, HPMC é solúvel em água e deve lixiviar para fora do filme de revestimento, criando poros cheios de água através dos quais o fármaco se difunde de maneira mais rápida que através das cadeias de EC (GUNDER et al., 1995). Os polímeros não biodegradáveis também são utilizados como integrantes do núcleo dos comprimidos e podem exercer várias funções. Como 
exemplo, o polímero hidroxipropil celulose pode ser utilizado como agente ligante nos processos de granulação úmida em leito fluidizado e pode interferir na taxa de liberação do fármaco (DESAl et al., 2006). Esses polímeros, como o HPMC, também podem funcionar como um sistema de matriz em comprimidos para a liberação controlada do fármaco (CAO et al., 2005).

\section{NOVAS PERSPECTIVAS}

A ciência dos polímeros tem sido o eixo principal para o desenvolvimento de novos SLFs nas últimas décadas. Avanços futuros na ciência dos polímeros deverão ser baseados em modificações das propriedades químicas e físicas dos polímeros, e em novas combinações de copolímeros com objetivos e componentes que podem liberar uma ampla variedade de agentes bioativos (PILLAI; PANCHAGNULA, 2001). Além disso, novos processos de fabricação e manipulação como marcadores moleculares (ALLENDER et al., 2000), tecnologia de fluido supercrítico (GHADERI et al., 2000) e engenharia em escala nanométrica têm promovido uma verdadeira revolução no desing, desenvolvimento e eficiência dos SLFs à base de polímeros.

FONTE DE FINANCIAMENTO: FINEP, FUNAPE, CAPES e CNPq.

\section{REFERÊNCIAS BIBLIOGRÁFICAS}

ALLENDER, C.J.; RICHARDSON, C.; WOODHOUSE, B.; HEARD, C.M.; BRAIN, K.R. Pharmaceutical applications for molecularly imprinted polymers. Int. J. Pharm., Amsterdam, v. 195, p. 39-43, 2000.

ANGELOVA, N.; HUNKELER, D. Rationalizing the design of polymeric biomaterials. Trends Biotechnol., Amsterdam , v. 17, p. 409-421, 1999.

BROCCHINI, S.; DUNCAN, R. Pendant drugs, release from polymers. In Encyclopaedia of Controlled Drug Delivery, v. 2. Edited by Mathowitz E. New York: John Wiley and Sons, 1999, p. 786-816.

CAO, Q.-R.; CHOI, Y.-W.; CUI, J.-H.; LEE, B.-J. Formulation, release characteristics and bioavailability of novel monolithic bydroxypropylmethylcellulose matrix tablets containing acetaminophen. J. Control. Release, Amsterdam, v. 108, p. 351-361, 2005.

CHANDRA, R.; RUSTGI, R. Biodegradable polymers. Prog. Polym. Sci., New York, v. 23, p. 1273-1335, 1998.

DESAI, D.; RINALDI, F.; KOTHARI, S.; PARUCHURI, S.; LI, D.; LAI, M.; FUNG, S. BOTH, D. Effect of hydroxypropyl cellulose (HPC) on dissolution rate of hydrochlorothiazide tablets. Int. J. Pharm., Amsterdam, v. 308, p. 40-45, 2006.

FU, K.; PACK, D.W.; KILBANOV, A.M.; LANGER, R. Visual evidence of acidic environment within degrading poly(lactic-co-glycolic acid)(PLGA) microspheres. Pharm. Res., New York, v. 17, p. 100-106, 2000.

GALAEV, I.Y.; MATTIASSON, B. "Smart" polymers and what they could do in biotechnology and medicine. Trends Biotechnol., Amsterdam, v. 17, p.335-340, 1999.

GHADERI, R.; ARTURSSON, P.; CARLFORS, J. A new method for preparing biodegradable microparticles and entrapment of hidrocortisone in DL-PLG microparticles using supercritical fluid. Eur. J. Pharm. Sci., Amsterdam, v. 10, p. 1-9, 2000.

GUNDER, W .; LIPPOLD, B.H.; LIPPOLD, B.C. Release of drugs from ethyl cellulose microcapsules (diffusion pellets) with pore formers and pore fusion, Eur. J. Pharm. Sci., Amsterdam, v. 3, p. 203-214, 1995.

JACKSON, K.; YOUNG, D.; PANT, S. Drug-excipient interactions and their affect on absorption. PSTT, v. 3, n. 10, p. 336-345, 2000.

JACOBS, I.C.; MASON, N.S. Polymer delivery systems concepts, in Polymeric delivery systems: properties and applications, B.A. Charpentier, Editor, American Chemical Society: Washington, DC. 1993, p. 1-17.

JENQUIN, M.R.; MCGINITY, J.W. Characterization of acrylic resin matrix films and mechanisms of drug-polymer interactions. Int. J. Pharm., Amsterdam, v. 101, n. 1, p. 23-34, 1994.

KUMAR, M.N.V.; KUMAR, N. Polymeric controlled drug delivery systems: perspective issues and opportunities. Drug dev. Ind. Pharm., New York, v. 27, p. 1-30, 2001.

LANGER, R.; PEPPAS, N.A. Advances in Biomaterials, Drug Delivery, and Bionanotechnology. AIChE J., New York, v. 49, n. 12, p. 2990-3006, 2003. 
LECOMTE, F.; SIEPMANN, J.; WALTHER, M.; MACRAE, R.J.; BODMEIER, R. Blends of enteric and GIT-insoluble polymers used for film coating: physicochemical characterization and drug release patterns. J. Control. Release, Amsterdam, v. 89, p. 457-471, 2003.

LI, S; VERT, M. Biodegradable polymers: polyesters. In Encyclopaedia of Controlled Drug Delivery. Edited by Mathowitz E. New York: John Wiley and Sons; 1999, v. 1, p. 71-93.

MANO, E.B.; MENDES, L.C. Introdução a Polímeros. 2. ed. revista e ampliada. São Paulo: Edgard Blücher, 1999, 191p.

MAO, H.Q.; KDAIYALA, I.; LEONG, K.W.; ZHAO, Z.; DANG, W. Biodegradable polymers: poly (phosphoester)s. In Encyclopaedia of Controlled Drug Delivery. Edited by Mathowitz E. New York: John Wiley and Sons; 1999, v. 1, p. 45-60.

PALMIERI, G.F.; BONACUCINA, G.; DI MARTINO, P.; MARTELLI, S. Microencapsutation of semisolid ketoprofen/polymer microspheres, Int. J. Pharm., Amsterdam, v. 242, p. 175-178, 2002.

PILLAI, O.; PANCHAGNULA, R. Polymers in drug delivery, Current Opinion in Chemical Biology, London, v. 5, p. 447-451, 2001.

RIOS, M. Polymers for Controlled Release: Formulation Follows Function. Pharm. Technol., New York, v. 29, n. 6, p. 42-50, 2005.

SCHWENDEMAN, S.P.; CARDAMONE, M.; BRANDON, M.R.; KLIBANOV, A.; LANGER, R. Stability of proteins and their delivery from biodegradable polymer microparticles, in: Microparticulate Systems for the Delivery of Proteins and Vaccines, Marcel Dekker, NewYork, 1996, pp. 1-49.

SJOBLOM, B. Method to obtain microparticles. /US Patent n. 20046753014/ (22 de junho de 2004).

UHRICH, K.E.; CANNIZZARO, S.M.; LANGER, R.S.; SHAKESHEFF, K.M. Polymeric systems for controlled drug release. Chem. Rev., Washington, v. 99, p. 3181-3198, 1999.

ZHU, Y. Properties of polymeric drug delivery systems prepared by hot-melt extrusion. 2002. Tese (Doutorado)Faculty of the Graduate School of the University of Texas, Austin, 2002. 